\title{
CONSEQUÊNCIAS DO ISOLAMENTO SOCIAL PARA A POPULAÇÃO IDOSA: UMA BREVE REVISÃO
}

Vitória Machado Barchinski; Universidade Católica de Pelotas; vitoriabarchinski19@gmail.com Sophia Link Pascotto; Universidade Católica de Pelotas; sophiapascotto@ymail.com Michelle Huber Fontana;Universidade Católica de Pelotas ; mitchfontana@hotmail.com Raquel Prado Thomaz; Hospital Universitário de Santa Maria; raquelpthomaz@gmail.com

\section{RESUMO}

Introdução: Em 2019, iniciou-se um surto de infecção por um novo coronavírus o qual rapidamente se tornou um problema de saúde pública global. Diante da pandemia se evidencia preocupação no que tange o isolamento social e suas consequências à população idosa. Os idosos são uma população de risco para adquirir e manifestar as formas graves da Covid-19 devido frequentemente serem portadores de múltiplas comorbidades. $\mathrm{O}$ isolamento, apesar de necessário e extremamente relevante, pode trazer impactos negativos. Objetivo: Avaliar as consequências do isolamento social para os idosos. Método: Trata-se de uma breve revisão sistematizada da literatura utilizando as bases de dados PubMed e National Institutes of Health, utilizando-se os seguintes descritores: "Elderly" AND "Covid-19". Foram identificados cerca de 80 estudos, dos quais foram selecionados 5 por se enquadrarem no tema. Resultados: Desses estudos, 4 deles elencaram a solidão como uma das principais consequências psicológicas do isolamento prolongado, um deles evidenciou o aumento do risco de declínio cognitivo. Um artigo destacou que, infelizmente, a falta de visitas familiares aumentou a vulnerabilidade dos moradores de ILPIs ao abuso e à negligência. E, um dos artigos avaliou como discriminatório o isolamento seletivo. Por fim, 3 deles incentivaram a utilização da tecnologia como forma de manter a comunicação e interação com os idosos. Conclusão: Apesar de necessário, o isolamento social pode trazer consequências cognitivas e psicossociais consideráveis. Portanto, é fundamental uma avaliação individualizada de cada idoso a fim de identificar os efeitos e sintomas advindos desse isolamento e utilizar as ferramentas tecnológicas para tentar minimizá-los.

Palavras-chave: Covid-19; Idoso; Isolamento; 